\title{
THE FU ORIONIS BINARY SYSTEM RNO 1B/1C
}

\author{
STUART MCMULDROCH AND GEOFFrey A. BLAKE \\ Division of Geological and Planetary Sciences, 170-25, California Institute of Technology, Pasadena, California 91125 \\ Electronic mail: sxm@sol1.gps.caltech.edu, gab@csardas.gps.caltech.edu
}

\section{ANNEILA I. SARGENT}

Division of Physics, Mathematics, and Astronomy, California Institute of Technology, 105-24, Pasadena, California 91125 Electronic mail: afs@mmstar.caltech.edu

Received 1994 June 8; revised 1994 September 19

\begin{abstract}
Observations of $\mathrm{CS}(7 \rightarrow 6)$ emission reveal a $\geqslant 3 \mathscr{C}_{\odot}$ core, $1.8 \times 10^{4} \mathrm{AU}$ in size, surrounding the FU Orionis binary system RNO 1B/1C. Fractional chemical abundances, calculated from LVG and LTE codes, are mostly similar to those in the cold core TMC 1 . However, values for $\mathrm{SiO} / \mathrm{H}_{2}$ and $\mathrm{CH}_{3} \mathrm{OH} / \mathrm{H}_{2}$ are enhanced, possibly by sputtering reactions or grain-grain collisions in the outflow associated with the young stars. Aperture synthesis maps of the 2.6 and $3.1 \mathrm{~mm}$ continuum emission at $\sim 5^{\prime \prime}$ and $\sim 9^{\prime \prime}$ resolution, respectively, reveal that RNO $1 \mathrm{C}$ is surrounded by a flattened, dusty envelope, $\sim 5000 \mathrm{AU}$ in size, with mass $\geqslant 1.1 \mathscr{C}_{\odot}$. High spatial resolution $\left(\sim 3^{\prime \prime}\right)$ interferometer observations of CS $(2 \rightarrow 1)$ emission may trace the dense walls of an outflow cavity comprised of two concentric arcs with dynamical ages of $4 \times 10^{3}$ and $1 \times 10^{4} \mathrm{yr}$. The velocity structure of lower density gas imaged in the $\mathrm{CO}(1 \rightarrow 0)$ transition is consistent with the arcs being formed by two energetic FU Orionis outbursts. Each event may have imparted more than 4 $\mathscr{C}_{\odot} \mathrm{km} \mathrm{s}^{-1}$ to the outflow, implying outburst mass loss rates of $\sim 10^{-4} \mathscr{M}_{\odot} \mathrm{yr}^{-1}$. It appears that RNO 1C is probably the driving source for the outflow and that, while pre-main sequence stars are in the FU Orionis stage, outbursts may dominate both outflow morphology and energetics.
\end{abstract}

\section{INTRODUCTION}

FU Orionis objects (FUors) may represent a pivotal stage in the evolution of solar mass pre-main sequence stars. They are characterized by a sudden increase in visual brightness of 5-6 mag with a much slower decline, and possess distinctive spectral signatures; the $\mathrm{H}_{\alpha}$ and $\mathrm{Na}$ I D lines exhibit P Cygni profiles with a suppressed emission component, while absorption linewidths and spectral type vary with wavelength and often show a "double-peaked" structure (Herbig 1977, 1989; Hartmann \& Kenyon 1987; Welty et al. 1992; Hartmann et al. 1993). FUors also display distinctive arcuate reflection nebulae and large infrared excesses (Goodrich 1987). Many of their observed properties have been interpreted in terms of an elevated rate of accretion through a circumstellar disk onto a T Tauri star (Hartmann \& Kenyon 1985, 1987). The number of detected FUor outbursts suggests the phenomenon is repetitive, with stars in the T Tauri stage of evolution undergoing up to 100 outbursts over $\sim 10^{6} \mathrm{yr}$ (Herbig 1977, 1989; Hartmann 1992). This hypothesis is supported by observations of outflows from young stellar objects (Reipurth 1992; McMuldroch et al. 1993).

The main core of the dark cloud L1287, located some 850 pc distant, contains the cometary nebula GN 00.33.9 (Yang et al. 1991). Within this nebula lies the proposed binary FUor system, RNO 1B and RNO 1C (Staude \& Neckel 1991; Kenyon et al. 1993). The binary components are separated by $6^{\prime \prime}$ at PA $\sim 45^{\circ}$. Staude \& Neckel (1991) first identified the heavily extincted RNO 1B $\left(A_{v} \sim 9 \mathrm{mag}\right)$ as a FUor and suggested that a nearby bright knot that they designated RNO 1C might contain an even more deeply embedded star. This knot was subsequently identified as a FUor (Kenyon et al. 1993). Both objects lie within the positional error bars of IRAS $00338+6312\left(L \sim 680 L_{\odot}\right)$ and are associated with water masers (Fiebig et al. 1992).

Single dish ammonia observations reveal a rotating core, $\sim 0.5 \times 0.2 \mathrm{pc}$ in size and elongated along PA $135^{\circ}$, around the FUors (Estalella et al. 1993). Maps in the CO $(1 \rightarrow 0)$, $\mathrm{HCO}^{+}(1 \rightarrow 0)$, and $\mathrm{CO}(3 \rightarrow 2)$ transitions indicate that the driving source(s) for an energetic bipolar outflow, oriented at $\mathrm{PA} \sim 45^{\circ}$ and situated within $30^{\circ}$ of the plane of the sky, lie within this core (Snell et al. 1990; Yang et al. 1991; Evans et al. 1994). Infrared polarimetric imaging (Weintraub \& Kastner 1993) suggests that the source of the outflow is closer to the nominal position of IRAS $00338+6312$ than RNO 1B or RNO 1C. A VLA map of the $3.6 \mathrm{~cm}$ continuum emission also displays a peak within 1 " of the IRAS position and has been interpreted as confirming the presence of a driving source quite distinct from the FUors (Anglada et al. 1994).

Although $\sim 7^{\prime \prime}$ resolution aperture synthesis images of CS $(2 \rightarrow 1)$ show an intensity maximum near the IRAS position, they lack the sensitivity and spatial resolution to delineate structure reliably (Yang et al. 1993). To clarify the detailed structure of the L1287 core, and as part of a larger project to examine FUor characteristics (McMuldroch et al. 1993), we have undertaken a program of sensitive high resolution millimeter and submillimeter observations using the Owens Valley millimeter array and the Caltech Submillimeter Observatory, the results of which we describe below. 
TABLE 1. CSO observations.

\begin{tabular}{|c|c|c|c|c|c|c|c|c|c|c|c|}
\hline $\begin{array}{c}\text { (1) } \\
\text { Molecule }\end{array}$ & $\begin{array}{c}(2) \\
\text { Transition }\end{array}$ & $\begin{array}{c}(3) \\
\nu \\
(\mathrm{GHz})\end{array}$ & $\begin{array}{c}(4) \\
\text { Peak T } \\
(\mathrm{K})\end{array}$ & $\begin{array}{c}(5) \\
\text { FWHM } \\
\left(\mathrm{kms}^{-1}\right)\end{array}$ & $\begin{array}{c}(6) \\
\text { Velocity } \\
\left(\mathrm{kms}^{-1}\right)\end{array}$ & $\begin{array}{c}(7) \\
\int T d v \\
\left(\mathrm{~K} \mathrm{kms}^{-1}\right)\end{array}$ & $\begin{array}{c}(8) \\
\text { Wings }\end{array}$ & $\begin{array}{c}(9) \\
\mathrm{N} \\
\left(\mathrm{cm}^{-2}\right)\end{array}$ & $\begin{array}{c}(10) \\
F_{C^{18} O^{1}}\end{array}$ & $\begin{array}{l}(11) \\
\mathrm{F}_{C O^{2}}\end{array}$ & $\begin{array}{c}(12) \\
\mathrm{F}_{T M C 1}\end{array}$ \\
\hline $\mathrm{SiO}$ & $5 \rightarrow 4$ & 217.105 & 0.3 & 2.9 & -15.7 & 0.7 & $?$ & $1(12)$ & $1(-11)$ & $5(-10)$ & $2(-12)$ \\
\hline $\mathrm{H}_{2} \mathrm{CO}$ & $3_{0,3} \rightarrow 2_{0,2}$ & 218.222 & 3.1 & 4.1 & -17.6 & 14.0 & blue & $1(14)$ & $1(-9)$ & $5(-8)$ & $2(-8)$ \\
\hline $\mathrm{CH}_{3} \mathrm{OH}$ & $4_{2} \rightarrow 3_{1}$ & 218.440 & 0.71 & 3.1 & -17.4 & 2.3 & $?$ & $4(14)$ & $4(-9)$ & $2(-7)$ & $2(-9)$ \\
\hline $\mathrm{H}_{2} \mathrm{CO}$ & $3_{2,2} \rightarrow 2_{2,1}$ & 218.475 & 0.6 & 3.4 & -16.9 & 2.6 & $?$ & $2(14)$ & $2(-9)$ & $1(-7)$ & $2(-8)$ \\
\hline $\mathrm{C}^{18} \mathrm{O}$ & $2 \rightarrow 1$ & 219.560 & 4.96 & 3.34 & -18.3 & 20.0 & no & $2(16)$ & $2(-7)$ & - & $2(-7)$ \\
\hline SO & $5_{6} \rightarrow 4_{5}$ & 219.949 & 1.5 & 2.7 & -17.2 & 6.0 & blue & $7(13)$ & $7(-10)$ & $4(-8)$ & $5(-9)$ \\
\hline${ }^{13} \mathrm{CO}$ & $2 \rightarrow 1$ & 220.399 & 15.9 & 3.30 & -18.6 & 69.0 & blue & $1(17)$ & $1(-6)$ & $5(-5)$ & $1(-6)$ \\
\hline $\mathrm{CO}$ & $2 \rightarrow 1$ & 230.538 & 32.9 & - & - & - & strong & - & - & - & \\
\hline CS & $7 \rightarrow 6$ & 342.883 & 1.9 & 3.3 & -17.0 & 8.8 & weak & $2(14)$ & $2(-9)$ & $1(-7)$ & $1(-8)$ \\
\hline $\mathrm{CO}$ & $3 \rightarrow 2$ & 345.796 & 25.8 & - & - & - & strong & - & - & - & \\
\hline $\mathrm{HCN}$ & $4 \rightarrow 3$ & 354.505 & 2.5 & 5.2 & -16.4 & 15.5 & yes & $1(14)$ & $1(-9)$ & $5(-8)$ & $2(-8)$ \\
\hline $\mathrm{HCO}^{+}$ & $4 \rightarrow 3$ & 356.734 & 8.8 & - & - & - & yes & - & - & - & \\
\hline
\end{tabular}

Notes to TABLE 1

(1) The fractional abundance relative to $\mathrm{H}_{2}$ derived using the $\mathrm{C}^{18} \mathrm{O}$ column density. (2) The fractional abundance relative to $\mathrm{H}_{2}$ derived using the $\mathrm{CO}$ column density.

\section{OBSERVATIONS}

\subsection{CSO Observations}

Observations of the core of L1287, encompassing RNO 1B, RNO 1C, and IRAS $00338+6312$, were acquired with the Caltech Submillimeter Observatory (CSO) on Mauna Kea, Hawaii, in 1991 July. The facility 230 and $345 \mathrm{GHz}$ SIS heterodyne receivers were used in conjunction with the CSO 500 and $50 \mathrm{MHz}$ bandwidth acousto-optical spectrometers (Ellison \& Miller 1987; Ellison et al. 1989). Calibration was accomplished by the standard chopper wheel method, while main beam efficiencies were calculated to approximately $20 \%$ accuracy from measurements of Jupiter. At 230 and 345 $\mathrm{GHz}$, the beamwidths were $31^{\prime \prime}$ and $21^{\prime \prime}$ (FWHM), with main beam efficiencies of 0.52 and 0.58 , respectively. All temperatures quoted in the paper have been corrected for these main beam efficiencies. Position switching to a reference position $10^{\prime}$ west of RNO 1B produced the least contaminated baselines. Pointing was checked regularly on planets and nearby point sources; absolute positions are accurate to within $10^{\prime \prime}$, relative positions within the maps to $\sim 5^{\prime \prime}$.

Several molecular species were detected towards RNO 1B in the 230 and $345 \mathrm{GHz}$ bands and are listed in Table 1. Spectra were acquired for the $\mathrm{CO}(2 \rightarrow 1), \mathrm{C}^{18} \mathrm{O}(2 \rightarrow 1)$, SiO $(5 \rightarrow 4)$, SO $\left(5_{6} \rightarrow 4_{5}\right), \mathrm{HCN}(4 \rightarrow 3), \mathrm{CH}_{3} \mathrm{OH}\left(4_{2} \rightarrow 3_{1}\right), \mathrm{HCO}^{+}$ $(4 \rightarrow 3), \mathrm{H}_{2} \mathrm{CO}\left(3_{0,3} \rightarrow 2_{0,2}\right)$, and $\mathrm{H}_{2} \mathrm{CO}\left(3_{2,2} \rightarrow 2_{2,1}\right)$ rotational transitions. In addition, ${ }^{13} \mathrm{CO}(2 \rightarrow 1)$ emission at $220 \mathrm{GHz}$ was mapped at half beam spacings over a region $\sim 45^{\prime \prime} \times 45^{\prime \prime}$ in size. More extensive maps, $\sim 80^{\prime \prime} \times 80^{\prime \prime}$, also sampled at half beam spacings, were made of the $\mathrm{CO}(3 \rightarrow 2)$ and $\mathrm{CS}$ $(7 \rightarrow 6)$ emission. Due to their small Einstein A coefficients, $\mathrm{CO}$ and ${ }^{13} \mathrm{CO}$ trace relatively cold, low density gas while $\mathrm{CS}$, with its higher dipole moment, delineates higher density gas.

\subsection{OVRO Observations}

Aperture synthesis mapping of the $J=1 \rightarrow 0$ transition of ${ }^{12} \mathrm{CO}$ and the $J=2 \rightarrow 1$ transition of CS at $\nu=115.271$ and
97.981 GHz, respectively, was carried out using the Owens Valley Radio Observatory (OVRO) millimeter-wave array between 1991 April and 1993 May. Observations at 115.272 $\mathrm{GHz}$ employed three $10.4 \mathrm{~m}$ diameter telescopes while the 97.981 GHz maps utilized four telescopes. Each telescope was equipped with an SIS receiver, cryogenically cooled to 4 $\mathrm{K}$, producing typical overall system temperatures of $520 \mathrm{~K}$ (SSB) at $115 \mathrm{GHz}$ and $320 \mathrm{~K}(\mathrm{SSB})$ at $98 \mathrm{GHz}$.

During the $\mathrm{CO}$ observations, two filter banks of $32 \times 1$ $\mathrm{MHz}$ and $32 \times 50 \mathrm{kHz}$ provided velocity resolutions of 2.6 and $0.13 \mathrm{~km} \mathrm{~s}^{-1}$. The CS spectral line observations used a digital correlator configured to provide velocity resolutions of 1.53 and $0.38 \mathrm{~km} \mathrm{~s}^{-1}$. In both instances, the bandpass was centered on the cloud velocity of $-18.3 \mathrm{~km} \mathrm{~s}^{-1}$ (Torrelles et al. 1983), while simultaneous continuum observations were obtained in a broadband channel of effective width $\sim 375 \mathrm{MHz}$

The phase and amplitude gains were calibrated by observing the quasar $0212+735$ at 25 min intervals, and the absolute flux scale was established from measurements of Neptune and Uranus. Maps were generated from calibrated visibility data using the NRAO AIPS software package. Positional accuracy is \pm 2 ". Maximum unprojected baselines of $140 \mathrm{~m}$ north-south and $200 \mathrm{~m}$ east-west gave a uniform weighted synthesized beam of $3.8 \times 2$ ". 7 , PA $90^{\circ}$, at $98 \mathrm{GHz}$ and a naturally weighted beam of $7.8 \times 4.8$, PA $85^{\circ}$, at 115 $\mathrm{GHz}$. For these beams, $T_{B}=1 \mathrm{~K}$ corresponds to 0.41 and $0.08 \mathrm{Jy} /$ beam. The phase center of the maps is $\alpha$ $(1950)=00^{\mathrm{h}} 33^{\mathrm{m}} 52.11^{\mathrm{s}}, \delta(1950)=+63^{\circ} 12^{\prime} 24^{\prime \prime} 0$, the position of RNO 1B (Staude \& Neckel 1991).

\section{CSO RESULTS AND ANALYSIS}

Table 1 lists the line parameters and integrated fluxes of molecular species observed toward RNO $1 \mathrm{~B} / 1 \mathrm{C}$ with the CSO. Dashes indicate where spectra are seriously affected by self-absorption or outflow. The presence of wing emission is 


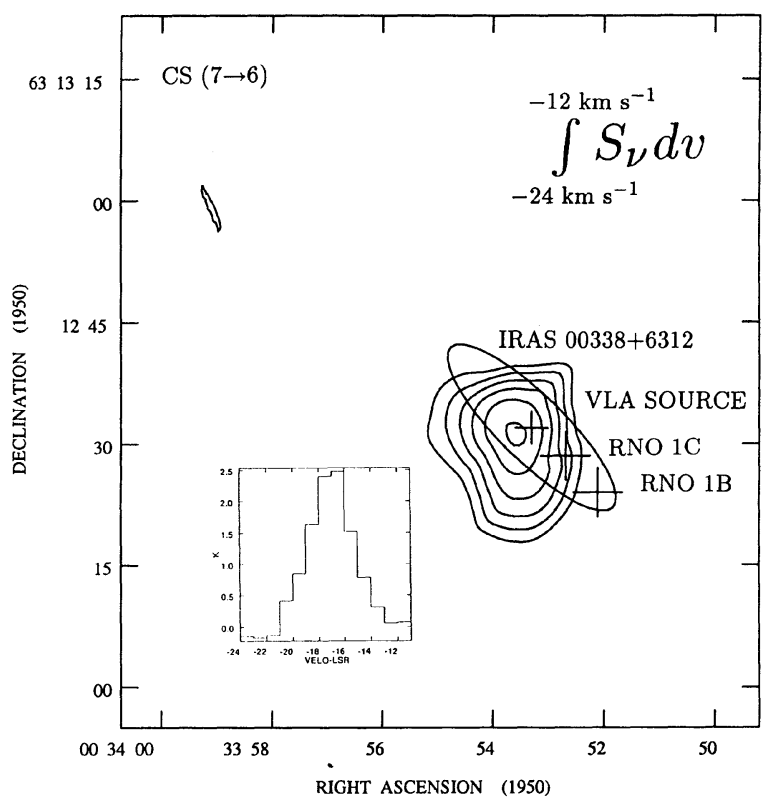

FIG. 1. A map of the CS $(7 \rightarrow 6)$ emission integrated between $V_{\mathrm{LSR}}=-24$ to $-12 \mathrm{~km} \mathrm{~s}^{-1}$. Contours begin at $8 \mathrm{~K}$ and are separated by $1 \mathrm{~K}$. The inset shows the CS spectrum in the region of peak emission. A cross 4 " in size marks the position of the VLA source while an ellipse indicates the range of positional uncertainty for IRAS $00338+6312$. Crosses $6^{\prime \prime}$ in size mark the positions of RNO 1B and RNO 1C.

indicated in column 8 , where a question mark indicates too low a signal-to-noise ratio to detect outflow signatures. The velocity of peak flux is indicated in column 6; molecules with low critical densities, such as $\mathrm{CO}$ and its isotopes, peak at $\sim-18.3 \mathrm{~km} \mathrm{~s}^{-1}$, while species with higher critical densities have maxima between -17.6 and $-15.7 \mathrm{~km} \mathrm{~s}^{-1}$. Where collisional cross sections were available, column densities and fractional abundances were calculated using an LVG code. Otherwise, an LTE code in the optically thin regime was employed.

\subsection{The Cloud Core}

Contours in Fig. 1 show the CS $(7 \rightarrow 6)$ emission integrated over the velocity range -24 to $-12 \mathrm{~km} \mathrm{~s}^{-1}$. Contours begin at $20 \%$ of the peak flux of $13 \mathrm{~K} \mathrm{~km} \mathrm{~s}^{-1}$ and are separated by $10 \%$. Emission is marginally resolved in the $21^{\prime \prime}$ beam. The peak is displaced $\sim 4^{\prime \prime}$ southwest of the IRAS position and $\sim 12$ " northwest of RNO 1C. As the spectrum inset in Fig. 1 shows, the line peaks at a velocity of -17.0 $\mathrm{km} \mathrm{s}^{-1}$. Excitation of the CS $(7 \rightarrow 6)$ transition requires densities in excess of $10^{6} \mathrm{~cm}^{-3}$, so that the observations trace a high density core surrounding the FUors displaced from the bulk cloud velocity of -18.3 by $1.3 \mathrm{~km} \mathrm{~s}^{-1}$. We therefore adopt $-17.0 \mathrm{~km} \mathrm{~s}^{-1}$ as the systemic velocity of the core containing the FUors.

The spectra of most species, apart from the $\mathrm{CO}$ isotopes, also peak near $-17.0 \mathrm{~km} \mathrm{~s}^{-1}$ implying an origin within the CS core. Calculations utilizing an LVG code and the ratio of the $\mathrm{H}_{2} \mathrm{CO}$ line pair indicate a kinetic temperature, $T_{\text {kin }}$, of $30-40 \mathrm{~K}$. At these temperatures, the ratio is only weakly dependent on density, making this a particularly useful temperature diagnostic (cf. Jansen et al. 1993; van Dishoeck et al. 1993a, b; Mangum \& Wootten 1993). LTE calculations yield similar values, $30-50 \mathrm{~K}$. Columns 10 and 11 in Table 1 list the range of fractional abundances for each molecular species, determined by dividing species column density by the molecular hydrogen column density of the cloud and of the outflow, respectively. In column 10 , lower limits are derived by dividing by the molecular hydrogen column density estimated from $\mathrm{C}^{18} \mathrm{O}$ measurements which trace the total mass in the core and surrounding cloud, assuming a canonical abundance $\mathrm{C}^{18} \mathrm{O} / \mathrm{H}_{2}=2 \times 10^{-7}$ (Chackerian \& Tipping 1983). In column 11, upper limits are derived by dividing by the molecular hydrogen column density estimated from the CO $(3 \rightarrow 2)$ wing column density of $2 \times 10^{17} \mathrm{~cm}^{-2}$, using ${ }^{12} \mathrm{CO} / \mathrm{H}_{2}=10^{-4}$ (Chackerian \& Tipping 1983) and assuming that emission originates entirely from outflowing gas. For comparison, column 12 lists fractional abundances for the cold core TMC 1 (van Dishoeck et al. 1993a, b, and references therein). In calculating the core mass, we adopt a CS abundance ratio typical of dense cores and intermediate between our two derived extremes of $\mathrm{CS} / \mathrm{H}_{2}=1 \times 10^{-8}$ (Irvine et al. 1987). For $T_{\mathrm{kin}}=35 \mathrm{~K}$, we calculate a core column density of $2.0 \times 10^{22} \mathrm{~cm}^{-2}$ in the optically thin limit, leading to a core mass, $\mathscr{C}_{\text {core }}$, of $2.6 \mathscr{H}_{\odot}$.

Figure 2 displays the ${ }^{12} \mathrm{CO},{ }^{13} \mathrm{CO}$, and $\mathrm{C}^{18} \mathrm{O}(2 \rightarrow 1)$ spectra at the position of RNO $1 \mathrm{~B}$. The $\mathrm{CO}\left(\right.$ and $\mathrm{HCO}^{+}$) spectrum shows a self-absorption minimum centered at -17.0 $\mathrm{km} \mathrm{s}^{-1}$, suggesting absorption is associated with a lower excitation cocoon surrounding the core seen in the CS $(7 \rightarrow 6)$ map. This feature is not observed in lower spatial resolution CO spectra (Yang et al. 1991), consistent with a localized absorbing layer.

\subsection{Cloud Characteristics}

Due to their low critical densities, $n_{\text {crit }} \sim 10^{3} \mathrm{~cm}^{-3}$, emission from the $J=2 \rightarrow 1$ transition of ${ }^{13} \mathrm{CO}$ and $\mathrm{C}^{18} \mathrm{O}$ molecules probably traces the ambient, larger-scale cloud structure. In both species, the spectra peak near $-18.3 \mathrm{~km} \mathrm{~s}^{-1}$. Linewidths are about $2.7 \mathrm{~km} \mathrm{~s}^{-1}$ away from RNO $1 \mathrm{~B} / 1 \mathrm{C}$, increasing to $3.4 \mathrm{~km} \mathrm{~s}^{-1}$ in the direction of the FUors. From the ratio of ${ }^{13} \mathrm{CO} / \mathrm{C}^{18} \mathrm{O}$ peak brightness temperatures, we estimate the $\mathrm{C}^{18} \mathrm{O}$ optical depth, $\tau_{\mathrm{C}^{18} \mathrm{O}}$, is 0.4 . LVG calculations, using the $\mathrm{C}^{18} \mathrm{O}$ line parameters $42^{\prime \prime}$ away from the core center, yield a cloud column density of $5 \times 10^{22} \mathrm{~cm}^{-2}$, assuming a standard abundance ratio of $\mathrm{C}^{18} \mathrm{O} / \mathrm{H}_{2}=2 \times 10^{-7}$ (Chackerian \& Tipping 1983).

\subsection{Outflow Characteristics}

In Fig. 2, the strong wing emission in ${ }^{12} \mathrm{CO}$ is not evident in $\mathrm{C}^{18} \mathrm{O}$, while ${ }^{13} \mathrm{CO}$ exhibits only a weak blueshifted wing. Comparison of $\mathrm{CO}$ and $\mathrm{C}^{18} \mathrm{O}$ spectra suggests inner velocity boundaries to the outflow wings of -20 and $-15 \mathrm{~km} \mathrm{~s}^{-1}$, excluding velocities effected by self-absorption, and similar to estimates based on $\mathrm{CO}(3 \rightarrow 2)$ measurements at $21^{\prime \prime}$ resolution (Evans et al. 1994). To investigate the outflow further, we mapped the $\mathrm{CO}(3 \rightarrow 2)$ line at $10^{\prime \prime}$ spacings over the region where wings were clearly evident. Emission was de- 


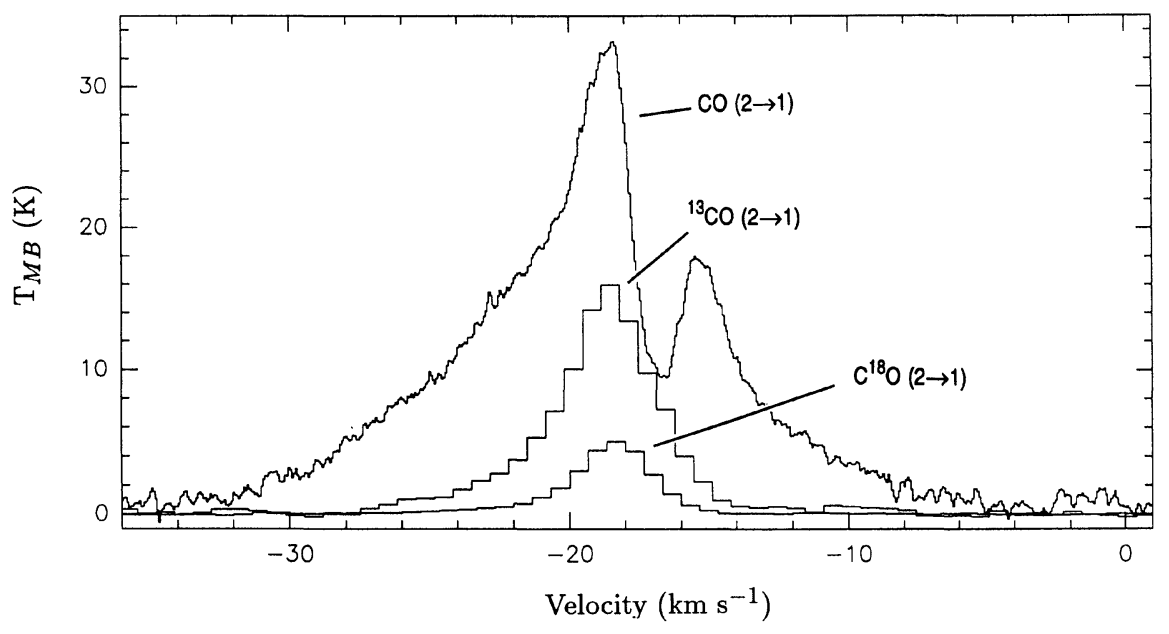

FIG. 2. Line emission in $\mathrm{CO},{ }^{13} \mathrm{CO}$, and $\mathrm{C}^{18} \mathrm{O}(2 \rightarrow 1)$ as detected at the $\mathrm{CSO}$. Temperatures have been corrected for main beam efficiencies.

tected only between -37 and $-3 \mathrm{~km} \mathrm{~s}^{-1}$ to a $3 \sigma$ limit of 0.9 $\mathrm{K}$. Self-absorption is clearly evident between -19.5 and $-15 \mathrm{~km} \mathrm{~s}^{-1}$. In Fig. 3, broken contours depict the blueshifted CO $(3 \rightarrow 2)$ component integrated over the velocity range -37 to $-20 \mathrm{~km} \mathrm{~s}^{-1}$. Solid contours delineate the weaker redshifted emission integrated between -15 and -3 $\mathrm{km} \mathrm{s}^{-1}$. Taken together, the blue and redshifted CO $(3 \rightarrow 2)$ emission regions trace a bipolar outflow extending $\sim 90^{\prime \prime}$ tip to tip along $\mathrm{PA} \sim 45^{\circ}$. The morphology is similar to that observed in CO $(1 \rightarrow 0)$ maps (Snell et al. 1990; Yang et al. $1991)$ and in the less completely sampled CO $(3 \rightarrow 2)$ obser-

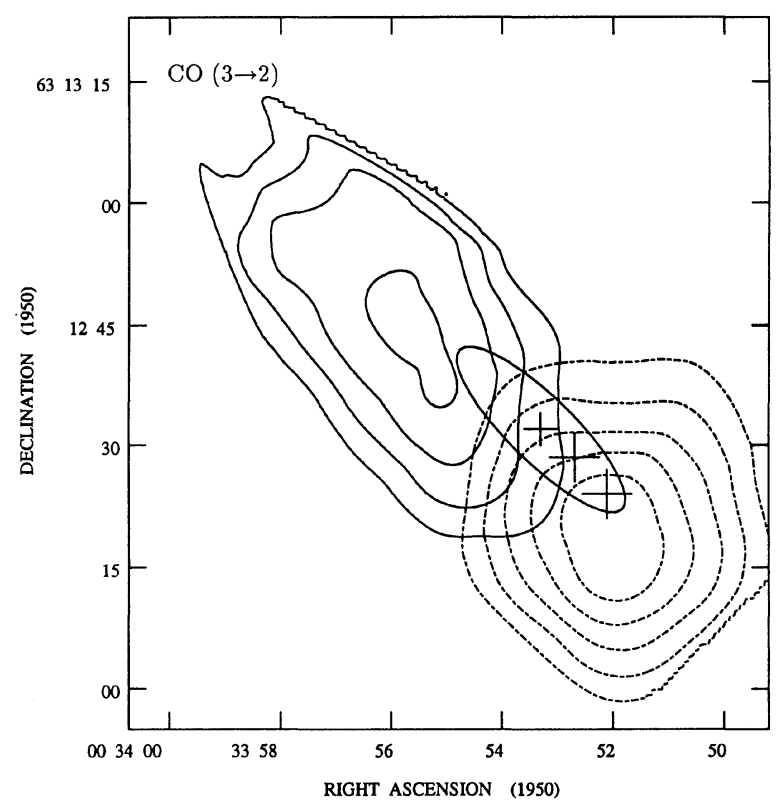

FIG. 3. A map of the CO $(3 \rightarrow 2)$ emission observed at the CSO. Broken contours depict the blueshifted emission, peak $149 \mathrm{~K} \mathrm{~km} \mathrm{~s}^{-1}$, integrated between velocities of -37 and $-20 \mathrm{~km} \mathrm{~s}^{-1}$. Solid contours depict the redshifted emission, peak $114 \mathrm{~K} \mathrm{~km} \mathrm{~s}^{-1}$, integrated between velocities -15 and $-3 \mathrm{~km} \mathrm{~s}^{-1}$. In both cases, contours begin at $50 \mathrm{~K} \mathrm{~km} \mathrm{~s}^{-1}$ and are spaced by $20 \mathrm{~K} \mathrm{~km} \mathrm{~s}^{-1}$. vations of Evans et al. (1994). The positions of IRAS 00338 +6312 , RNO 1C, and RNO 1B are located between the redshifted and blueshifted emission peaks, implying, as expected, that one of them is the driving source of the outflow.

Following Scoville et al. (1986), we calculate the mass in each outflow lobe from the equations

$$
\begin{aligned}
\int N(\mathrm{CO}) d \Omega= & 4.75 \times 10^{12} \frac{\left(T_{x}+0.93\right)}{e^{-22.34 / T_{x}}} \\
& \times \frac{\tau_{\mathrm{CO}}}{\left(1-e^{\left.-\tau_{\mathrm{CO}}\right)}\right.} \iint T_{\mathrm{MB}} d v d \Omega
\end{aligned}
$$

and

$$
\mathscr{C}=4.04 \times 10^{9} \frac{\mu m_{\mathrm{H}_{2}}\left(D_{\mathrm{kpc}}\right)^{2} \int N(\mathrm{CO}) d \Omega}{X(\mathrm{CO})} \mathscr{C}_{\odot} .
$$

Here, $\iint T_{\mathrm{MB}} d v d \Omega$ is the integrated line intensity summed over the solid angle $d \Omega$ for which emission is apparent, and has values of $2.1 \times 10^{-6}$ and $3.6 \times 10^{-6}$ $\mathrm{K} \mathrm{km} \mathrm{s}^{-1} \mathrm{sr}^{-1}$ for the blue and red wings, respectively. $T_{x}$ is the excitation temperature; $\tau$ is the optical depth; $\mu$ is the mean molecular weight; $m_{\mathrm{H}_{2}}$ is the mass of molecular hydrogen; $D$ is the source distance in kiloparsecs, and $X(\mathrm{CO})$ is the $\mathrm{CO}$ abundance relative to hydrogen. We adopt $X(\mathrm{CO})=10^{-4}$ (Chackerian \& Tipping 1983) and an average excitation temperature of $15 \mathrm{~K}$, the typical kinetic temperature in the outflow. A $5 \mathrm{~K}$ temperature difference corresponds to an approximate $20 \%$ change in mass. Optical depths of 9.2 and 3.1 for the blue and red wings are calculated from the $\mathrm{CO} /{ }^{13} \mathrm{CO}(3 \rightarrow 2)$ brightness ratio (Evans et al. 1994), with resulting masses of 1.4 and $0.5 \mathscr{C b}_{\odot}$. Using flux weighted average outflow velocities of -6.5 and $+3.5 \mathrm{~km} \mathrm{~s}^{-1}$ relative to the systemic velocity, we estimate the momenta in the blue and red lobes to be 9.1 and $1.8 \mathscr{C}_{\odot} \mathrm{km} \mathrm{s}^{-1}$. 

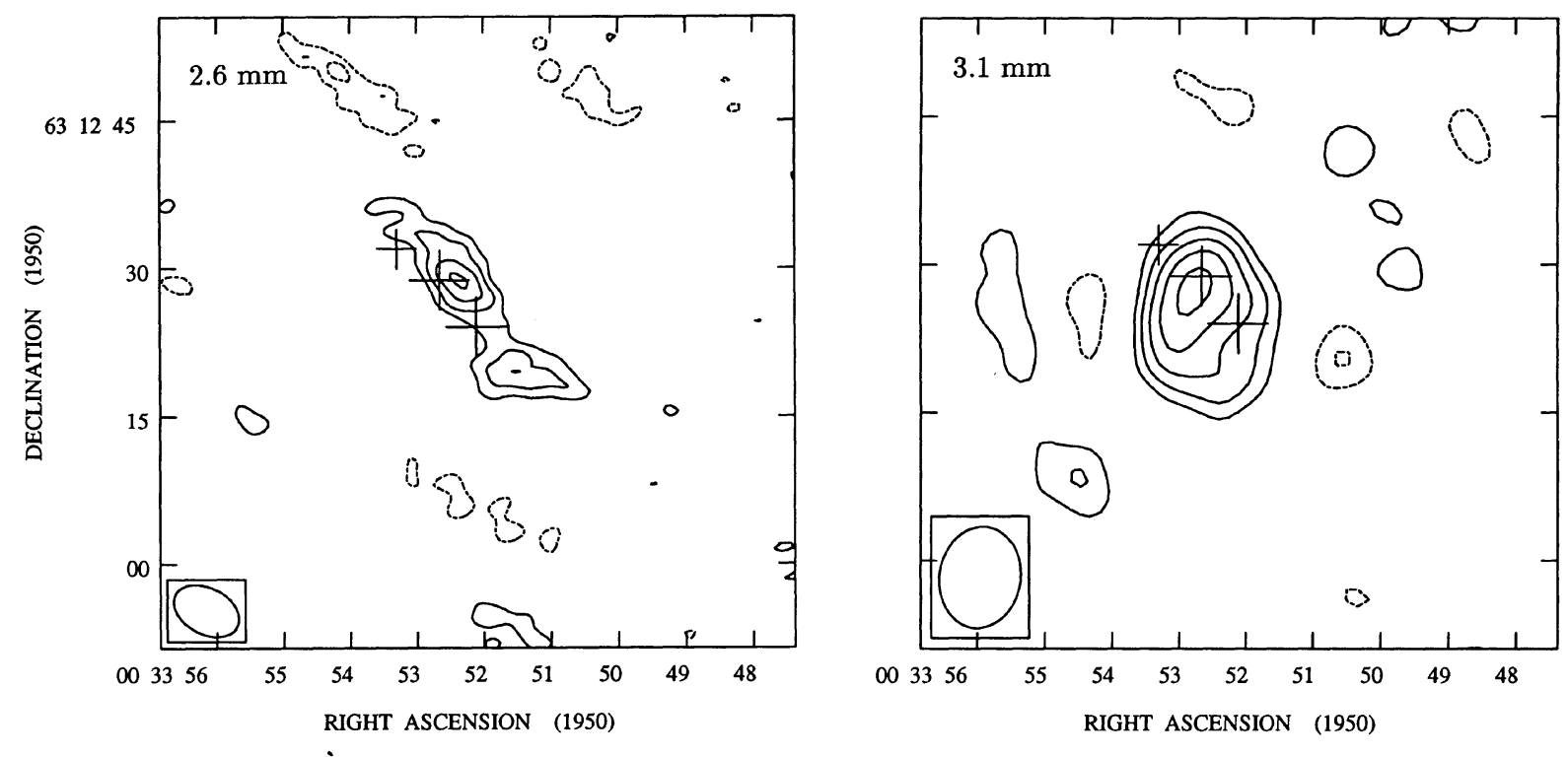

FIG. 4. Aperture synthesis maps of the 2.6 and $3.1 \mathrm{~mm}$ continuum emission observed toward RNO $1 \mathrm{~B} / 1 \mathrm{C}$. Contours begin at the $2 \sigma$ level of 11 and 9 $\mathrm{mJy} / \mathrm{beam}$ at 2.6 and $3.1 \mathrm{~mm}$, respectively, and are thereafter separated by $1 \sigma$. An ellipse in the lower left corner denotes the size of the synthesized beam in all OVRO maps. In this and all subsequent maps, the error ellipse of the IRAS source position is omitted to minimize confusion.

\section{OWENS VALLEY RESULTS AND ANALYSIS}

\subsection{Continuum Emission in the Core}

Figures 4(a) and 4(b) show maps of the 2.6 and $3.1 \mathrm{~mm}$ continuum flux in the vicinity of RNO 1B/1C. Extended flux is at low signal to noise levels at $3.1 \mathrm{~mm}$ and contaminated by sidelobes at $2.6 \mathrm{~mm}$. Morphological differences between the two maps are strongly affected by the beam pattern since emission in the central core is only marginally resolved in each case, with a 7."0 $044^{\prime \prime} 0\left(\mathrm{PA} 37^{\circ}\right)$ beam at $2.6 \mathrm{~mm}$ and a $10^{\prime \prime} 4 \times 8.2\left(\mathrm{PA}-8^{\circ}\right)$ beam at $3.1 \mathrm{~mm}$. Within the positional errors of \pm 2 ", both the $2.6 \mathrm{~mm}$ peak of $26 \mathrm{mJy} / \mathrm{beam}$ and the $3.1 \mathrm{~mm}$ peak of $29 \mathrm{mJy} /$ beam coincide with RNO $1 \mathrm{C}$. This strongly suggests that emission from RNO $1 \mathrm{C}$ dominates the emission pattern. High resolution maps of millimeter continuum emission are probably the most reliable way of identifying embedded thermal sources (cf. Sargent \& Welch 1994).

VLA observations at $\lambda=3.6$ and $6 \mathrm{~cm}$ revealed a source near the IRAS position with spectral index 1.5, typical of ionized gas (McCutcheon et al. 1991; Anglada et al. 1994). Extrapolation indicates that such free-free processes contribute less than $6 \mathrm{mJy}$ at $\lambda=3.1 \mathrm{~mm}$. Our aperture synthesis maps therefore trace thermal dust emission. The concentration of dust towards RNO $1 \mathrm{C}$ suggests that this source is possibly the primary driver of the outflow.

Following Hildebrand (1983), the mass of molecular gas associated with RNO $1 \mathrm{C}$ can be calculated from the 2.6 and $3.1 \mathrm{~mm}$ flux densities of $60 \pm 30$ and $45 \pm 20 \mathrm{mJy}$, respectively, using

$$
\mathscr{M}_{\mathrm{H}_{2}}=\frac{0.120 F_{\mathrm{Jy}}\left(D_{\mathrm{kpc}}\right)^{2}\left(\lambda_{\mathrm{mm}}\right)^{3}\left(e^{14.4 / \lambda_{\mathrm{mm}} T_{d}}-1\right)}{\kappa_{\nu}} \mathscr{M}_{\odot},
$$

where $F_{\mathrm{Jy}}$ is the observed continuum flux density in Janskys, $\lambda_{\mathrm{mm}}$ is the observing wavelength in millimeters, $T_{d}$ is the dust temperature, and $\kappa_{\nu}$ is the mass opacity coefficient for the dust grains at frequency $\nu$. We assume a characteristic dust temperature of $50 \mathrm{~K}$, slightly larger than our value for the core kinetic temperature of $35 \mathrm{~K}$. Estimates of $\kappa_{\nu}$ can vary by over an order of magnitude. Here we adopt

$$
\kappa_{\nu}=\kappa_{0}\left(\frac{0.25 \mathrm{~mm}}{\lambda_{\mathrm{mm}}}\right)^{\beta} \text {, }
$$

with $\kappa_{0}=0.1 \mathrm{~cm}^{2} \mathrm{gr}^{-1}$ (Hildebrand 1983) and $\beta \sim 1$ (cf. Beckwith \& Sargent 1991). Resulting masses are 1.1 \pm 0.6 and $1.4 \pm 0.6 \mathscr{U}_{\odot}$ at 2.6 and $3.1 \mathrm{~mm}$. This value of $\kappa_{\nu}$ is conservatively high, giving lower limits to the mass surrounding RNO $1 \mathrm{C}$. Upper limits to the mass, using the same coefficients, at the positions of RNO $1 \mathrm{~B}$ and the VLA source are 0.3 and $0.4 \mathscr{l}_{\odot}$, at 2.6 and $3.1 \mathrm{~mm}$, respectively.

\subsection{CO $(1 \rightarrow 0)$ Maps}

Figures $5(\mathrm{a})-5(\mathrm{~d})$ show aperture synthesis CO $(1 \rightarrow 0)$ line maps of the RNO $1 \mathrm{C}$ region at $\sim 5^{\prime \prime}$ resolution in the velocity ranges -44.2 to $-31.2,-31.2$ to $-23.4,-23.4$ to -18.2 , and -15.6 to $-5.2 \mathrm{~km} \mathrm{~s}^{-1}$. No flux was detected outside these velocity ranges to a $3 \sigma$ limit of $240 \mathrm{mJy} / \mathrm{beam}$. At the highest blueshifted velocities, Fig. 5(a), the peak flux is centered between RNO 1C and RNO 1B; at smaller blueshifted velocities, Figs. 5(b) and 5(c), emission extends away from the stars to the southwest. At low redshifted velocities seen in Fig. 5(d), emission peaks to the northeast of RNO $1 \mathrm{C}$. Taken together, these features form the blue and redshifted 

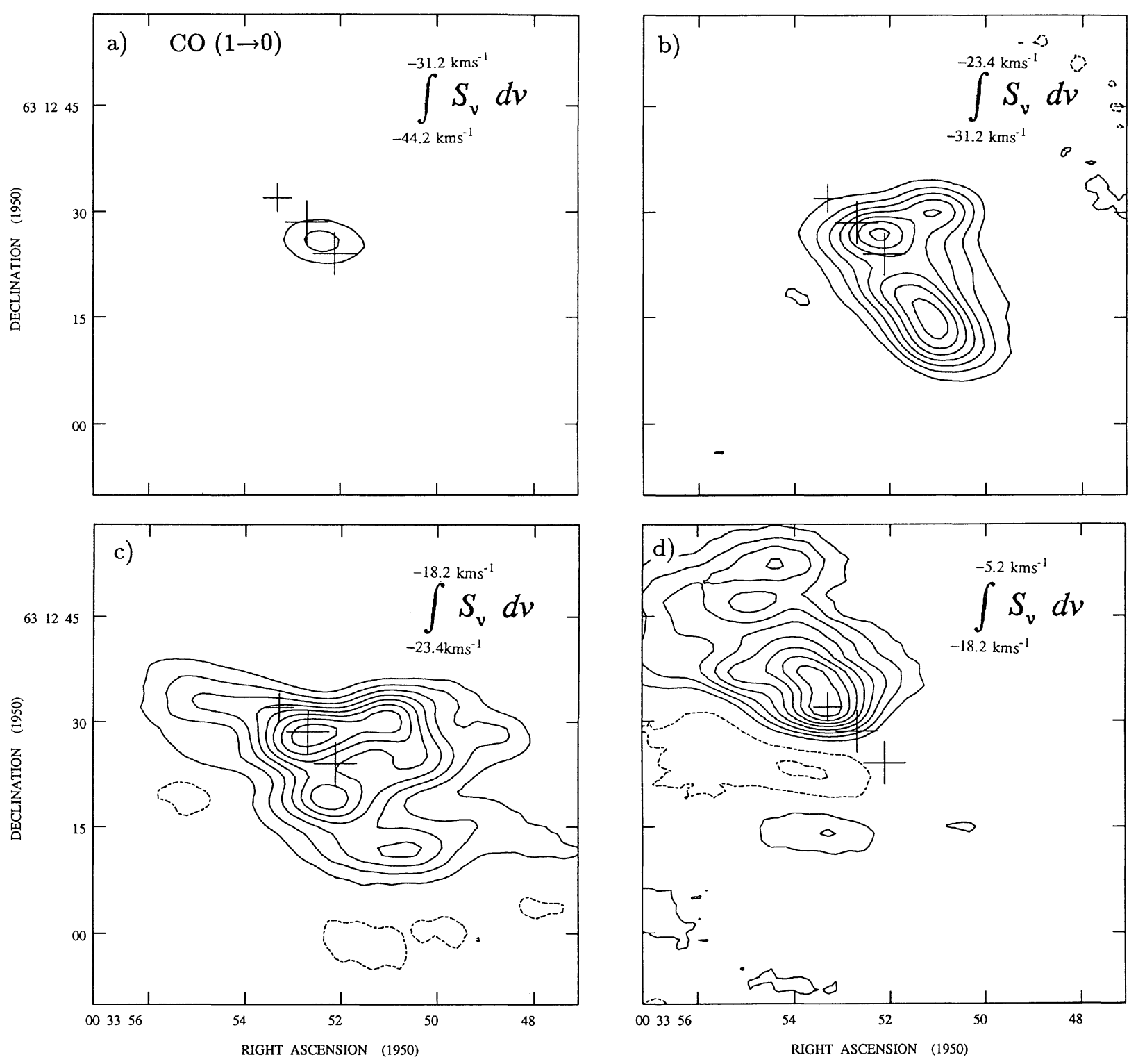

FIG. 5. Maps of the CO $(1 \rightarrow 0)$ emission observed by the OVRO integrated over the velocity ranges -44.2 to $-31.2,-31.2$ to $-23.4,-23.4$ to -18.2 , and -18.2 to $-5.2 \mathrm{~km} \mathrm{~s}^{-1}$. Contours begin at and are separated by $0.5 \mathrm{Jy} / \mathrm{beam} \mathrm{km} \mathrm{s}^{-1}$.

components of the bipolar outflow. In our CSO CO $(3 \rightarrow 2)$ maps, the blueshifted lobe also extends to the southwest and the redshifted lobe to the northeast, so that highly blueshifted CO $(1 \rightarrow 0)$ emission is expected southwest of the driving source, reaffirming the central role of RNO 1C. Indeed, as shown in Fig. 5(c), emission near the core velocity peaks within the positional uncertainties at RNO 1C. Strong extended emission lies along PA $115^{\circ}$, approximately perpendicular to the outflow. It is unclear from the present data if this is a disk-like structure or part of the outflow.

The total integrated fluxes for each of the regions depicted in Figs. 5(a)-5(d) are 15, 233, 401, and $339 \mathrm{Jy} \mathrm{km} \mathrm{s}^{-1}$, respectively. Following Scoville et al. (1986), the mass of $\mathrm{H}_{2}$ implied in each case is given by

$$
\begin{aligned}
\mathscr{l} b= & 2.19 \times 10^{-9} \frac{\left(T_{x}+0.93\right)}{e^{-5.53 / T_{x}}} \\
& \times \frac{\tau_{\mathrm{CO}}}{\left(1-e^{\left.-\tau_{\mathrm{CO}}\right)}\right.} \frac{D_{\mathrm{kpc}}^{2}}{X(\mathrm{CO})} \int S_{\nu} d v \mathscr{C}_{\odot},
\end{aligned}
$$

where $\int S_{\nu} d v$ is the integrated flux density in units Jy km s${ }^{-1}$ and the remaining parameters are as described for Eq. (1). As before, we adopt an excitation temperature for the outflow of $15 \mathrm{~K}$ and assume the emission is optically thin. We calculate minimum masses for the regions depicted in Figs. $5(\mathrm{a})-5(\mathrm{~d})$ of $5 \times 10^{-3}, 0.08,0.15$, and $0.12 \mathscr{l}_{\odot}$ and associated momenta of $0.12,0.85,0.55$, and $0.67 \mathscr{M b}_{\odot} \mathrm{km} \mathrm{s}^{-1}$, respectively. 

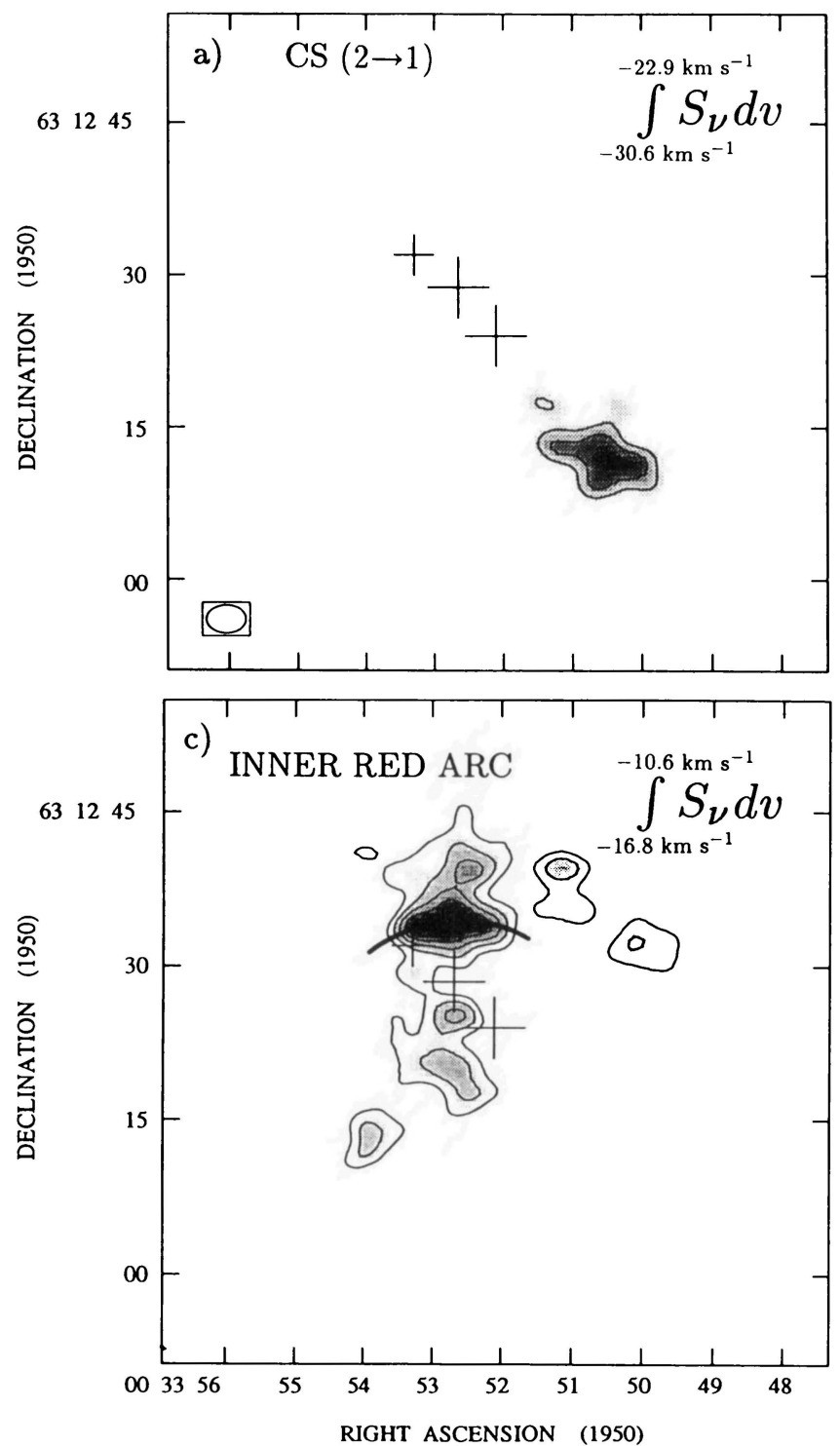
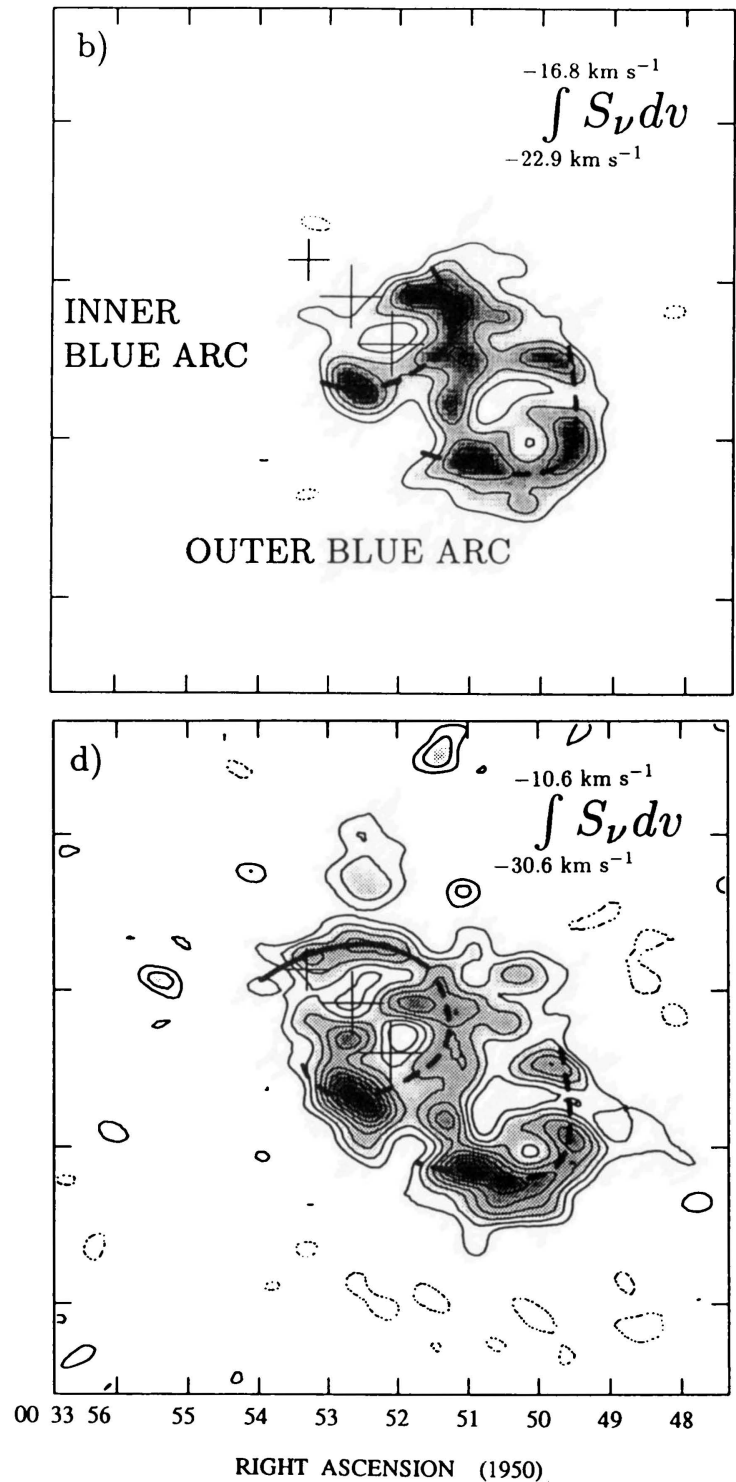

Fig. 6. Aperture synthesis maps of the CS $(2 \rightarrow 1)$ emission integrated over velocities -30.6 to $-22.9,-22.9$ to $-16.8,-16.8$ to -10.6 , and -30.6 to -10.6 $\mathrm{km} \mathrm{s}^{-1}$, respectively. Contours in each map begin at $20 \%$ of the peak total integrated flux, $3.42 \mathrm{Jy} / \mathrm{beam} \mathrm{km} \mathrm{s} \mathrm{s}^{-1}$, and are separated by $10 \%$. The greyscale image depicts the same emission for clarification. Heavy broken lines trace the blueshifted arcs described in the text while a solid line follows the redshifted arc.

\subsection{CS $(2 \rightarrow 1)$ Emission}

Emission from the CS $(2 \rightarrow 1)$ rotational transition was detected only between velocities of -30.6 and $-10.6 \mathrm{~km} \mathrm{~s}^{-1}$ to a $3 \sigma$ limit of $26 \mathrm{mJy} / \mathrm{beam}$. No high velocity gas corresponding to the $\mathrm{CO}(1 \rightarrow 0)$ emission seen in Fig. 5(a) was observed. The contour and greyscale aperture synthesis maps of Figs. $6(a)-6(c)$ show the CS integrated intensity at $\sim 3^{\prime \prime}$ resolution over the velocity ranges -30.6 to $-22.9,-22.9$ to -16.8 , and -16.8 to $-10.6 \mathrm{~km} \mathrm{~s}^{-1}$. Figure $6(\mathrm{~d})$ shows the total integrated CS $(2 \rightarrow 1)$ emission. The CS $(2 \rightarrow 1)$ emission peak is not coincident with the CS $(7 \rightarrow 6)$ maximum (Fig. 1 ), probably due to the lower resolution and greater positional uncertainties of the CSO data.

At the highest observed blueshifted velocities, Fig. 6(a), emission peaks $\sim 18^{\prime \prime}$ southwest of RNO $1 \mathrm{C}$ with a low intensity region extending back toward the star. The morphol- ogy is roughly analogous to that in the CO $(1 \rightarrow 0)$ map of Fig. 5(b). We infer the CS structure represents a denser component of the blueshifted outflow. In Fig. 6(b), a possible double arcuate structure lies to the southwest of the FUors; the more prominent arc is located about $25^{\prime \prime}$ from RNO $1 \mathrm{C}$ while a possible inner arc lies within $10^{\prime \prime}$. Redshifted emission shown in Fig. 6(c) peaks $\sim 5^{\prime \prime}$ north of RNO 1C and may be the redshifted counterpart of the inner arc seen in Fig. 6(b). Overall, the redshifted emission is less prominent than the blueshifted component. Indeed, our CSO observations demonstrate that the red outflow has a lower peak intensity and is slightly more extended so that it may be resolved out. Within $1 \mathrm{~km} \mathrm{~s}^{-1}$ of the systemic velocity, -17 $\mathrm{km} \mathrm{s}^{-1}$, emission also appears resolved out or possibly selfabsorbed.

Maps presented in Fig. 7 illustrate the behavior of blueshifted CS emission between -22.32 and $-18.11 \mathrm{~km} \mathrm{~s}^{-1}$ at 


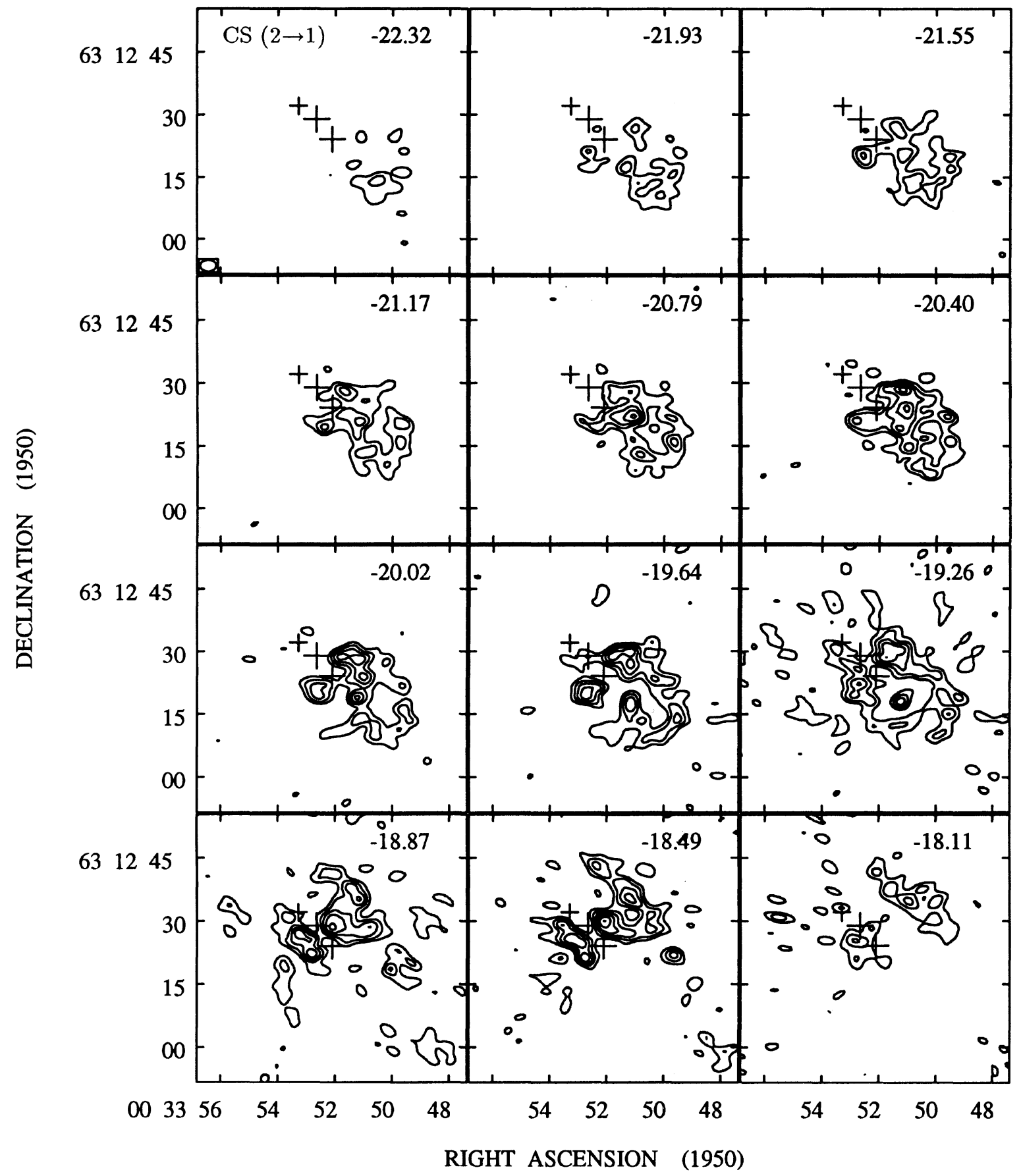

FIG. 7. High velocity resolution maps of the blueshifted CS $(2 \rightarrow 1)$ emission observed toward RNO $1 \mathrm{~B} / 1 \mathrm{C}$ between the velocity range -22.32 to -18.11 $\mathrm{km} \mathrm{s}^{-1}$. The velocity of each channel is indicated in the upper right corner. Contours begin at the $3 \sigma$ level, $270 \mathrm{mJy} / \mathrm{beam}$, and are thereafter separated by $2 \sigma$.

a velocity resolution of $0.38 \mathrm{~km} \mathrm{~s}^{-1}$. There is a condensation $25^{\prime \prime}$ southwest of RNO $1 \mathrm{C}$ at the highest velocities. Approaching the systemic velocity, $-17 \mathrm{~km} \mathrm{~s}^{-1}$, this bifurcates forming a loop. Emission is also present $\sim 10^{\prime \prime}$ to the south and west of RNO $1 \mathrm{C}$ and forms a possible inner arc between velocities of -21.55 and $-20.02 \mathrm{~km} \mathrm{~s}^{-1}$. At distances of $\sim 10^{\prime \prime}-25^{\prime \prime}\left(8.5-21.2 \times 10^{3} \mathrm{AU}\right)$ from RNO $1 \mathrm{C}$ the observed velocity displacements are not consistent with rotation or infall. We suggest emission arises from outflowing gas. Between -19.64 and $-18.49 \mathrm{~km} \mathrm{~s}^{-1}$, the morphology is dominated by a dumbbell structure oriented perpendicularly to the outflow. For these velocities which are closer to the systemic value, it is difficult to ascertain whether emission arises from rotation, infall, or outflow.

The mass of swept up gas can again be calculated (cf. Scoville et al. 1986; Ohashi et al. 1991) using 


$$
\begin{aligned}
\mathscr{C}= & 1.3 \times 10^{-11} \frac{\left(T_{x}+0.4\right)}{e^{-7.1 / T_{x}}} \\
& \times \frac{\tau_{\mathrm{CS}}}{\left(1-e^{\left.-\tau_{\mathrm{CS}}\right)}\right.} \frac{D_{\mathrm{kpc}}^{2}}{X(\mathrm{CS})} \int S_{\nu} d v \mathscr{M}_{\odot},
\end{aligned}
$$

where we use the same parameters as in for Eq. (5) but with a CS abundance ratio, $X(\mathrm{CS})$, of $1 \times 10^{-8}$ (Irvine et al. 1987). Assuming optically thin emission, we calculate masses of $0.24,1.20$, and $0.94 \mathscr{C b}_{\odot}$ for the structures observed in each of the velocity ranges of Figs. $6(\mathrm{a})-6(\mathrm{c})$. Estimated masses and momenta for the outer and inner "arcs" are 0.58 and $0.52 \mathscr{C}_{\odot}$, and 2.20 and $2.08 \mathscr{C}_{\odot} \mathrm{km} \mathrm{s}^{-1}$.

\section{DISCUSSION}

\subsection{Chemistry}

Our CSO maps demonstrate that the FU Orionis binary system, RNO $1 \mathrm{~B} / 1 \mathrm{C}$, is surrounded by a dense core, $1.8 \times 10^{4}$ $\mathrm{AU}$ in size, of mass $\sim 3 \mathscr{C}_{\odot}$. Although the core is only weakly offset from the bulk cloud velocity, outflowing gas is kinematically quite distinct from the ambient cloud material. Mass estimates of the quiescent and outflowing components are provided by the $\mathrm{C}^{18} \mathrm{O}$ and ${ }^{12} \mathrm{CO} J=2 \rightarrow 1$ lines, from which we derive the fractional abundance limits presented in Table 1. For comparison, this table also presents the abundances found in the TMC 1 dark cloud.

The abundances of most molecules, especially those not obviously associated with the outflow, are comparable to those in molecular cloud cores devoid of star formation. Certain species, notably $\mathrm{CS}, \mathrm{H}_{2} \mathrm{CO}$, and $\mathrm{SO}$ are weakly enhanced in the outflowing gas; by contrast, $\mathrm{CH}_{3} \mathrm{OH}$ and $\mathrm{SiO}$ show enhancements larger than an order of magnitude. While the small enrichments may result from density and temperature enhancements caused by the outflow, the enhancements of $\mathrm{SiO}$ and $\mathrm{CH}_{3} \mathrm{OH}$ are likely the result of chemical interactions.

Moderate enhancements in $\mathrm{SiO}$ and $\mathrm{CH}_{3} \mathrm{OH}$ are not unusual in regions of outflow activity (cf. Bachiller \& GomezGonzalez 1992; van Dishoeck et al. 1993a, b), where low velocity shocks can drive new reaction routes even for low luminosity sources. The refractory $\mathrm{SiO}$ molecule is most likely produced by sputtering or evaporation of dust grains in regions of directly shocked and entrained material. The high observed abundances of $\mathrm{CH}_{3} \mathrm{OH}$ towards $\mathrm{RNO} 1 \mathrm{~B} / 1 \mathrm{C}$ cannot be produced either by low temperature ion-molecule or gas phase shock chemistry (Sandell et al. 1994). However, grain mantle evaporation can provide the needed methanol (Blake et al. 1991); the increased velocity dispersion surrounding bipolar outflows may result in shorter grain-grain collision times at relatively low collision velocities, thereby increasing grain mantle evaporation rates without shattering the grains themselves (Blake et al. 1995). These two distinct processes of grain sputtering, as opposed to softer grain-grain collisions, should have different spatial distributions. However, higher spatial resolution observations are required to investigate the RNO 1B/1C morphology further.

\subsection{The Driving Source of the Outflow}

Aperture synthesis maps of the continuum emission at 2.6 and $3.1 \mathrm{~mm}$, shown in Figs. 4(a) and 4(b), suggest a lower limit of $1.1 \mathscr{C l}_{\odot}$ for the mass around RNO 1C. Reprocessed radiation from the dust may account for the sharp rise in the RNO 1B/1C spectral energy distribution beyond $12 \mu \mathrm{m}$ (Kenyon et al. 1993). For a source $\sim 5000-7000 \mathrm{AU}$ in size, a spherical dust distribution produces a visual extinction $A_{v} \geqslant 50$ mag (Spitzer 1978), much larger than the observed value of $A_{v} \sim 12 \mathrm{mag}$ (Staude \& Neckel 1991). It seems likely that the dust is asymmetrically distributed, but the large size precludes a truly centrifugally supported circumstellar accretion disk. Since continuum emission appears marginally resolved at different beam sizes, we suggest that the material surrounding RNO $1 \mathrm{C}$ follows a power law density distribution (Ladd et al. 1991; Terebey et al. 1993).

The location of RNO $1 \mathrm{C}$ at the peak of millimeter continuum emission, its central position between the peaks of the blue and redshifted outflow lobes [Figs. 5(a)-5(d)], and its association with the CS $(2 \rightarrow 1)$ dumbbell structure (Fig. 7), together indicate that the FUor is the driving source for the outflow in L1287. Infrared polarization measurements and centimeter continuum emission suggest a third more embedded source near the IRAS position is possibly the driving source (McCutcheon et al. 1991; Weintraub \& Kastner 1993; Anglada et al. 1994). However, the millimeter continuum peak is located $\sim 8^{\prime \prime}$ from the IRAS position, virtually excluding it as the outflow source, since millimeter continuum measurements are probably the most accurate determinant of the position of an embedded object. We suggest that the infrared observations are sensitive to the dust geometry surrounding RNO 1C and are therefore affected by the outflow cavity structure. Moreover, the radio emission spectral index of 1.5 is characteristic of free-free emission regions frequently observed at moderate distances from energetic, outflow sources (McMullin et al. 1994). The error in the IRAS position easily includes both RNO $1 \mathrm{~B}$ and RNO $1 \mathrm{C}$. It is difficult to reconcile the spatial coincidence of the IRAS source, the centimeter emission peak, and pattern of polarization with our new observations.

\subsection{Outflow Structure}

High spatial resolution $\left(3.8 \times 2.7\right.$, PA $\left.90^{\circ}\right)$ maps of the CS $(2 \rightarrow 1)$ emission reveal the high density structure of the L1287 outflow in Figs. 6(a)-6(d) and 7. We do not detect the axisymmetric offset in the outflow noted by Yang et al. (1993) and interpreted as evidence of helical structure. Overall, the emission pattern resembles a limb-brightened outflow cavity lying nearly in the plane of the sky. The morphology is consistent with CS emission tracing the cavity walls that have been compressed by the outflow wind and with the higher velocity $\mathrm{CO}$ emission tracing entrained low-density gas.

In Fig. 8(a), contours of the blueshifted $\mathrm{CO}(1 \rightarrow 0)$ emission integrated between velocities -44.2 and $-20.8 \mathrm{~km} \mathrm{~s}^{-1}$ are shown, while in Fig. 8(b), contours trace the redshifted $\mathrm{CO}$ emission integrated between -15.6 and $-5.2 \mathrm{~km} \mathrm{~s}^{-1}$. In both figures, a greyscale image shows the total integrated CS 

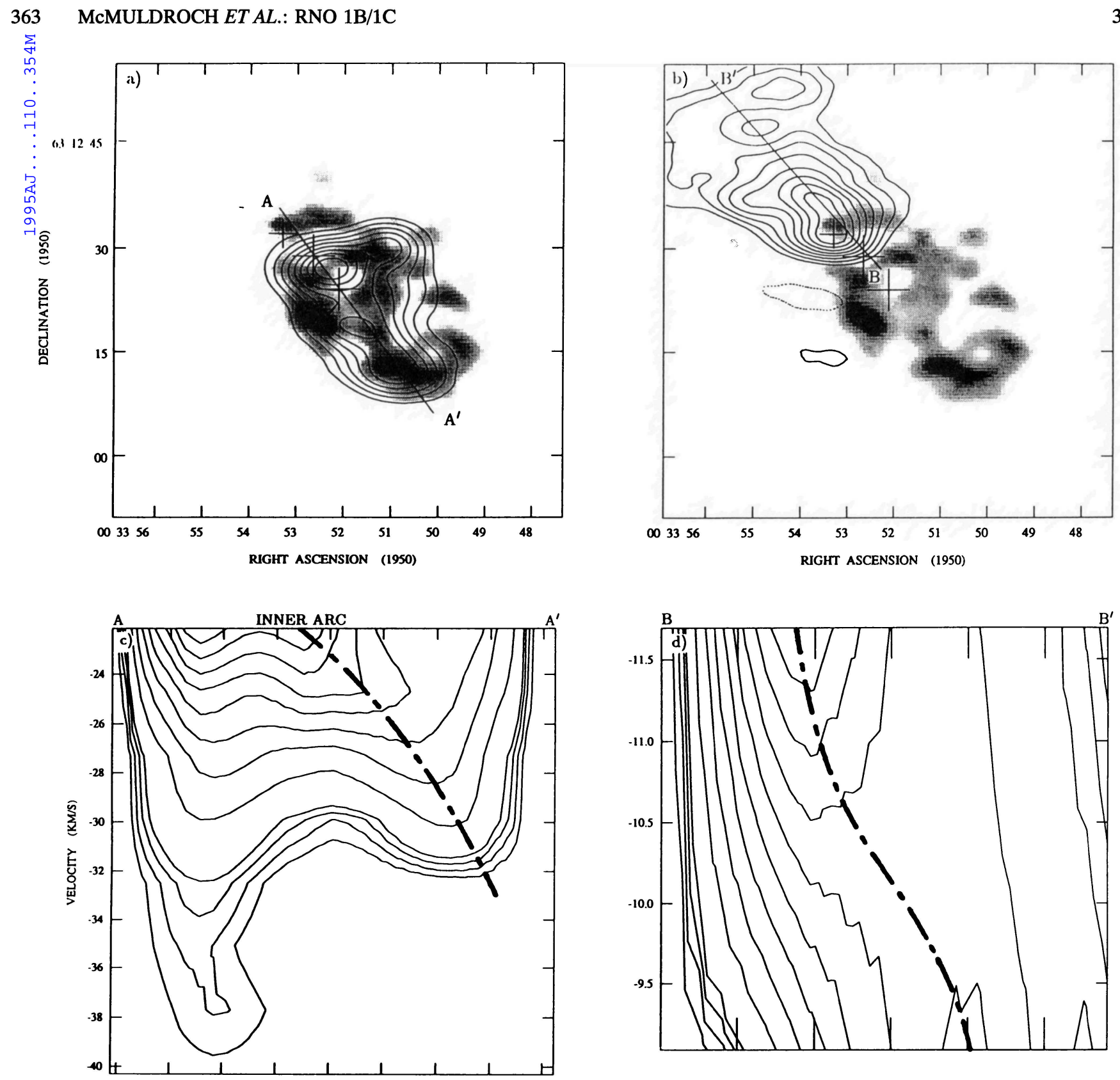

FIG. 8. Aperture synthesis maps of $\mathrm{CO}(1 \rightarrow 0)$ and CS $(2 \rightarrow 1)$ emission towards RNO 1B/1C. Contours in (a) depict the blueshifted CO emission integrated over velocities -44.2 to $-20.8 \mathrm{~km} \mathrm{~s}^{-1}$ while contours in (b) depict the redshifted CO emission integrated between -15.6 and $-5.2 \mathrm{~km} \mathrm{~s}^{-1}$. Contours start at $20 \%$ and are separated by $10 \%$ of the peak flux in each velocity interval. In both cases, the greyscale shows the total integrated CS emission. (c) and (d) are the position-velocity diagrams of emission along $\mathrm{AA}^{\prime}$ and $\mathrm{BB}^{\prime \prime}$, respectively. Contours are at $4 \%, 6 \%, 8 \%, 10 \%, 20 \%, 30 \%, 40 \%, 50 \%, 60 \%, 70 \%, 80 \%$, and $90 \%$ of the peak flux in each diagram. Heavy dashed lines trace the approximately linear velocity trends discussed in the text.

$(2 \rightarrow 1)$ emission which we believe traces the outflow cavity. The maximum blueshifted $\mathrm{CO}$ intensity occurs southwest of RNO 1C and extends to the southwest. Secondary peaks occur at the positions of the inner and outer arcs described in Sec. 4.3. By contrast, redshifted $\mathrm{CO}$ peaks at the position of the northern inner arc and extends to the northeast. Figures $8(\mathrm{c})$ and $8(\mathrm{~d})$ are position-velocity (PV) cuts through the blue and red lobes along lines $\mathrm{AA}^{\prime}$ and $\mathbf{B B}^{\prime}$, respectively. It is clear from Fig. 8(c), that gas over a wide velocity range contributes to the emission around RNO 1C labelled as Region 1. Across the southern inner arc, emission appears at velocities between $\sim-22$ and $-32 \mathrm{~km} \mathrm{~s}^{-1}$. Between the arcs, peak velocity linearly increases with distance from the source as marked by a dashed line. A similar although much weaker trend is apparent for the redshifted emission in Fig. 8(d).

Although outflow models predict the existence of cavities (Masson \& Chernin 1993; Raga \& Cabrit 1993; Chernin et al. 1994), few have been confirmed (Uchida et al. 1987; Moriarty-Schieven et al. 1987; Avery et al. 1990; MeyersRice \& Lada 1991). There is some suggestion that cavities around FUors may be more common (Moriarty-Schieven et al. 1987; McMuldroch et al. 1993). In terms of time scales and velocities characteristic of outflows, a FUor event is explosive and instantaneous. The inner and outer arcs traced in CS $(2 \rightarrow 1)$ may be interpreted as dense gas swept up by two separate FUor outbursts. Material is driven at all velocities by an outburst, which becomes velocity sorted with increas- 
ing distance from the source, leading to the PV structures seen in Figs. 8(a) and 8(b). Gas swept up by the more recent outburst lies close to the source at all velocities, while velocity sorting of the gas swept up earlier has produced the observed linear position-velocity relationship. Alternatively, the observed velocity pattern can be interpreted in terms of time-dependent collimated jet outflow models (Raga \& Biro 1993; Raga \& Cabrit 1993), in which internal working surfaces propagate along a collimated jet entraining gas in the wake of a bowshock. These models produce cavity morphologies comparable to those presented here and also yield radial velocities that increase away from the source. However, these models are for outflows with a time-dependent wind velocity rather than mass loss rate as is the case for FUors.

The momenta of the cavity's inner and outer arcs, estimated from measurements of CS $(2 \rightarrow 1)$ emission, are 2.0 and $2.1 \mathscr{M}_{\odot} \mathrm{km} \mathrm{s}^{-1}$, respectively, while the momenta of the CO $(1 \rightarrow 0)$ outflow lobes are 1.6 and $1.0 \mathscr{C}_{\odot} \mathrm{km} \mathrm{s}^{-1}$. A classical FUor outburst lasting $100 \mathrm{yr}$, with a wind velocity of $500 \mathrm{~km} \mathrm{~s}^{-1}$ and a mass loss rate of $10^{-4} \mathscr{C}_{\odot} \mathrm{yr}^{-1}$, imparts only $\sim 0.5 \mathscr{M}_{\odot} \mathrm{km} \mathrm{s}^{-1}$ of momentum. This suggests that if the arcs were formed by FUor activity then the outbursts were exceptionally energetic entraining $\sim 4 \mathscr{M}_{\odot} \mathrm{km} \mathrm{s}^{-1}$. The present luminosity of $L_{\text {bol }} \sim 1000 L_{\odot}$ for RNO $1 \mathrm{~B} / 1 \mathrm{C}$ suggests the current outburst is also exceptionally energetic, assuming wind energy scales with accretion luminosity (Cabrit \& Bertout 1992).

Single dish $\mathrm{CO}(3 \rightarrow 2)$ observations yield an outflow lifetime of $\sim 10^{4} \mathrm{yr}$. The velocity dispersions seen in Figs. 8(c) and $8(\mathrm{~d})$ suggest a repetition time scale of $4 \times 10^{3} \mathrm{yr}$, while dynamical age estimates of the arcs, made from CS observations assuming an inclination angle $i=30^{\circ}$, yield lower bounds of $4 \times 10^{3}$ and $1 \times 10^{4}$ yr. A maximum of three outbursts could have occurred in the lifetime of the outflow yielding $\sim 12 \mathscr{M}_{\odot} \mathrm{km} \mathrm{s}^{-1}$ of momentum, approximately the total outflow momentum of $11 \mathscr{M}_{\odot} \mathrm{km} \mathrm{s}^{-1}$ (see Sec. 3.3). If the arcs are indeed caused by FUor activity, then FUor outbursts possibly dominate both outflow morphology and outflow energetics in this source.

\section{CONCLUSIONS}

We have carried out sensitive high resolution millimeter and submillimeter observations of the FU Orionis binary system RNO 1B/1C. CS $(7 \rightarrow 6)$ measurements trace a dense core, $1.8 \times 10^{4} \mathrm{AU}$ in size, mass $\sim 3 \mathscr{M}_{\odot}$, at $V_{\mathrm{LSR}}=-17.0$ $\mathrm{km} \mathrm{s}^{-1}$. Fractional chemical abundances in this core, calculated from LVG and LTE codes, are largely similar to those in the cold core TMC 1. Enhancements in the $\mathrm{SiO}$ and $\mathrm{CH}_{3} \mathrm{OH}$ abundances are consistent with a low velocity shock liberating grain mantle material to the gas phase. Indeed, observations of the $\mathrm{CO}(3 \rightarrow 2)$ emission delineate a bipolar outflow, oriented at PA $45^{\circ}$, with blueshifted emission lying to the southwest of RNO $1 \mathrm{~B} / 1 \mathrm{C}$.

Aperture synthesis observations of 2.6 and $3.1 \mathrm{~mm}$ continuum emission from the RNO $1 \mathrm{~B} / 1 \mathrm{C}$ core at $7.0 \times 4$ ". 6 (PA $\left.62^{\circ}\right)$ and $10.4 \times 8.2\left(\mathrm{PA}-8^{\circ}\right)$ resolution lead us to conclude that RNO $1 \mathrm{C}$ is surrounded by a flattened dusty envelope, $\sim 5000 \mathrm{AU}$ in size, with a minimum mass of $1.1 \mathscr{M b}_{\odot}$. The morphology of the $\mathrm{CO}(1 \rightarrow 0)$ aperture synthesis images also suggests RNO $1 \mathrm{C}$ is possibly the driving source. A dumbbell structure oriented at $90^{\circ}$ to the outflow axis and $3^{\prime \prime}$ southwest of RNO $1 \mathrm{C}$ is visible in CS $(2 \rightarrow 1)$ images and further strengthens this interpretation.

The CS $(2 \rightarrow 1)$ interferometer maps are consistent with the presence of an outflow cavity lying nearly in the plane of the sky. Two concentric arcs, $8 \times 10^{3}$ and $8 \times 10^{3} \mathrm{AU}$ from RNO 1C, are apparent in blueshifted emission, although only the inner arc has a redshifted counterpart. The gas velocity structure is consistent with formation by two energetic FU Orionis outbursts, $4 \times 10^{3}$ and $1 \times 10^{4} \mathrm{yr}$ ago. The time between outbursts, $\sim 5 \times 10^{3} \mathrm{yr}$, is comparable to previous estimates based on source frequency statistics of $2 \times 10^{3} \mathrm{yr}$. Each outburst may have imparted $\geqslant 4 \mathscr{M}_{\odot} \mathrm{km} \mathrm{s}^{-1}$ to the outflow implying a mass loss rate of $\mathscr{C} \sim 10^{-4} \mathscr{C}_{\odot} \mathrm{yr}^{-1}$ for a 500 $\mathrm{km} \mathrm{s}^{-1}$ wind lasting $100 \mathrm{yr}$. It appears that while young premain sequence stars are in the FU Orionis stage, outbursts may possibly dominate both outflow morphology and energetics.

We are indebted to the staff of the Owens Valley millimeter-wave array and the Caltech Submillimeter Observatory for their unfailing support and extend special thanks to Taco for his assistance with the CSO mapping software. We are extremely grateful to Ewine van Dishoeck and Todd Groesbeck for providing use of their LVG and LTE codes. We extend our thanks to the referee for many helpful suggestions. Astronomy with the Owens Valley Array is funded by NSF Grant AST 93-14079 and the CSO by NSF Grant AST 93-13929. S.M. is supported by NASA Grants NAGW-2297 and NAGW-3140, part of the Origins of Solar Systems Research Program.

\section{REFERENCES}

Anglada, G., Rodriguez, L. F., \& Girart, J. M. 1994, ApJ, 420, L91 Avery, L. W., Hayashi, S. S., \& White, G. L. 1990, ApJ, 357, 524 Bachiller, R., \& Gomez-Gonzalez, J. 1992, A\&AR, 3, 257

Beckwith, S. V. W., \& Sargent, A. I. 1991, ApJ, 381, 250

Blake, D. F., Allamandola, L., Sandford, S., Hudgins, D., \& Freund, F. 1991, Science, 254, 548

Blake, G. A., Sandell, G., van Dishoeck, E. F., Groesbeck, T. D., Mundy, L. G., \& Aspin, C. 1995, ApJ, 441, 689

Cabrit, S., \& Bertout, C. 1992, A\&A, 261, 274

Chackerian, C., \& Tipping, R. H. 1983, J. Molec. Spectrosc., 99, 431
Charnley, S. B. 1994, ApJ (submitted)

Chernin, L., Masson, C., \& Pino, E. M. G. D. 1994, ApJ, 426, 204

Ellison, B. E., \& Miller, R. L. 1987, Int. J. Infrared and Millimeter Waves, 8,608

Ellison, B. E., Schaffer, P. L., Schaal, W., Vail, D., \& Miller, R. E. 1989, Int. J. Infrared and Millimeter Waves, 10, 937

Estalella, R., Mauersberger, R., Torrelles, J. M., Anglada, G., Gomez, J. G., Lopez, R., \& Muders, D. 1993, ApJ, 419, 698

Evans III, N. J., Balkum, S., Levreault, R. M., Hartmann, L., \& Kenyon, S. 1994, ApJ (in press) 
Fiebig, D., Duschl, W. J., \& Tscharnuter, W. M. 1992, IAU Circ. 5438, 1

Goodrich, R. W. 1987, PASP, 99, 116

Hartmann, L. 1992, in NATO ASI on The Formation and Evolution of Stars, edited by N. Kylafis and C. Lada

II Hartmann, L., \& Kenyon, S. J. 1985, ApJ, 299, 462

III Hartmann, L., \& Kenyon, S. J. 1987, ApJ, 312, 243

Hartmann, L., Kenyon, S. J., \& Hartigan, P. 1993, in Protostars and Planets

III, edited by E. H. Levy and J. I. Lunine (University of Arizona, Tucson) Herbig, G. H. 1977, ApJ, 217, 693

Herbig, G. H. 1989, in ESO Workshop on Low-Mass Star Formation and Pre-Main Sequence Objects, edited by B. Reipurth (ESO, Garching), p. 233

Hildebrand, R. H. 1983, QJRAS, 24, 267

Irvine, W. M., Goldsmith, P. F., \& Hjalmarson, ̊̊ 1987, in Interstellar Processes, edited by D. Hollenbach and H. A. Thronson (Reidel, Dordrecht) Jansen, D. J., van Dishoeck, E. F., \& Black, J. H. 1994, A\&A, 282, 605

Kenyon, S. J., Hartmann, L., Gomez, M., Carr, J. S., \& Tokunaga, A. 1993, AJ, 105, 1505

Ladd, E. F., Adams, F. C., Casey, S., Davidson, J. A., Fuller, G. A., Harper, D. A., Myers, P. C., \& Padman, R. 1991, ApJ, 382, 555

Mangum, J., \& Wootten, A. 1993, ApJS, 89, 123

Masson, C. R., \& Chernin, L. M. 1993, ApJ, 414, 230

McCutcheon, W. H., Dewdney, P. E., Purton, C. R., \& Sato, T. 1991, AJ, 101,1435

McMuldroch, S., Sargent, A. I., \& Blake, G. A. 1993, AJ, 106, 2477

McMullin, J. P, Mundy, L. G., Wilking, B. A., Hezel, T., \& Blake, G. A. 1994 , in preparation

Meyers-Rice, B. A., \& Lada, C. J. 1991, ApJ, 368, 445

Moriarty-Schieven, G. H., Snell, R. L., Strom, S. E., Schloerb, F. P., Strom, K. M., \& Grasdalen, G. L. 1987, ApJ, 319, 742
Ohashi, N., Kawabe, R., Hayashi, M., \& Ishiguro, M. 1991, AJ, 102, 2054 Raga, A. C., \& Biro, S. 1993, MNRAS, 264, 758

Raga, A., \& Cabrit, S. 1993, A\&A, 278, 267

Reipurth, B. 1992, A\&A, 257, 693

Sandell, G., Knee, L. B. G., Aspin, C., Robson, I. E., \& Russell, A. P. G. 1994, A\&A (submitted)

Sargent, A. I., \& Welch, W. J. 1993, ARA\&A, 31, 297

Scoville, N. Z., Sargent, A. I., Sanders, D. B., Claussen, M. J., Masson, C.

R., Lo, K. Y., \& Phillips, T. G. 1986, ApJ, 303, 416

Snell, R. L., Dickman, R. L., \& Huang, Y.-L. 1990, ApJ, 352, 139

Spitzer, L. 1978, Physical Processes in the Interstellar Medium (Wiley, New York)

Staude, H. J., \& Neckel, Th. 1991, A\&A, 244, L13

Terebey, S., Chandler, C. J., \& Andre, P. 1993, ApJ, 414, 759

Torrelles, J. M., Rodríguez, L. F., Cantó, J., Marcaide, J., \& Gyulbudaghian, A. L. 1983, RMxA, 8, 147

Uchida, Y., Kaifu, N., Shibata, K., Hayashi, S. S., Hasegawa, T., \& Hamatake, H. 1987, PASJ, 39, 907

van Dishoeck, E. F., Blake, G. A., Draine, B. T., \& Lunine, J. I. 1993a, in Protostars and Planets III, edited by E. H. Levy and J. I. Lunine (University of Arizona, Tucson)

van Dishoeck, E. F., Jansen, D. J., \& Phillips, T. G. 1993b, A\&A, 279, 541

Weintraub, D. A., \& Kastner, J. 1993, ApJ, 411, 767

Welty, A., Strom, S., Edwards, S., Kenyon, S. J., \& Hartmann, L. 1992, ApJ, 397, 260

Yang, J., Umemoto, T., Iwata, T., \& Fukui, Y. 1991, ApJ, 373, 137

Yang, J. Ohashi, N., \& Fukui, Y. 1993, in Second Northeast-Asian Regional Meeting on Recent Development in Millimeter-Wave and Infrared Astronomy, edited by S. H. Cho and H. S. Chung (Korea Astronomy Observatory, Daejon) 